University of Nebraska - Lincoln

DigitalCommons@University of Nebraska - Lincoln

2014

Testing metapopulation concepts: effects of patch characteristics and neighborhood occupancy on the dynamics of an endangered lagomorph

\author{
Mitchell J. Eaton \\ Phillip T. Hughes \\ James E. Hines \\ James D. Nichols
}

Follow this and additional works at: https://digitalcommons.unl.edu/usgsstaffpub

Part of the Geology Commons, Oceanography and Atmospheric Sciences and Meteorology Commons, Other Earth Sciences Commons, and the Other Environmental Sciences Commons

This Article is brought to you for free and open access by the US Geological Survey at DigitalCommons@University of Nebraska - Lincoln. It has been accepted for inclusion in USGS Staff -- Published Research by an authorized administrator of DigitalCommons@University of Nebraska - Lincoln. 


\title{
Testing metapopulation concepts: effects of patch characteristics and neighborhood occupancy on the dynamics of an endangered lagomorph
}

\author{
Mitchell J. Eaton, Phillip T. Hughes, James E. Hines and James D. Nichols \\ M. J. Eaton (meaton@usgs.gov), J. E. Hines and J. D. Nichols, Patuxent Wildlife Research Center, US Geological Survey, 12100 Beech Forest \\ Road, Laurel, MD 20708, USA. Present address for MJE: Southeast Climate Science Center, US Geological Survey, 127H David Clark Labs, \\ Dept of Biology, North Carolina State Univ., Raleigh, NC 27695, USA. - P. T. Hughes, National Key Deer Refuge, US Fish and Wildlife \\ Service, 28950 Watson Boulevard, Big Pine Key, FL 33043, USA.
}

\begin{abstract}
Metapopulation ecology is a field that is richer in theory than in empirical results. Many existing empirical studies use an incidence function approach based on spatial patterns and key assumptions about extinction and colonization rates. Here we recast these assumptions as hypotheses to be tested using 18 years of historic detection survey data combined with four years of data from a new monitoring program for the Lower Keys marsh rabbit. We developed a new model to estimate probabilities of local extinction and colonization in the presence of nondetection, while accounting for estimated occupancy levels of neighboring patches. We used model selection to identify important drivers of population turnover and estimate the effective neighborhood size for this system. Several key relationships related to patch size and isolation that are often assumed in metapopulation models were supported: patch size was negatively related to the probability of extinction and positively related to colonization, and estimated occupancy of neighboring patches was positively related to colonization and negatively related to extinction probabilities. This latter relationship suggested the existence of rescue effects. In our study system, we inferred that coastal patches experienced higher probabilities of extinction and colonization than interior patches. Interior patches exhibited higher occupancy probabilities and may serve as refugia, permitting colonization of coastal patches following disturbances such as hurricanes and storm surges. Our modeling approach should be useful for incorporating neighbor occupancy into future metapopulation analyses and in dealing with other historic occupancy surveys that may not include the recommended levels of sampling replication.
\end{abstract}

The importance of space and location to population dynamics was recognized by Wright $(1931,1940)$ in population genetics and later by Levins $(1969,1970)$ in population ecology. Levins (1969, 1970) introduced the term "metapopulation", which has become an important concept in population and conservation ecology. In particular, the metapopulation concept emphasizes the relevance of space, the movement of organisms, and turnover (local extinctions and colonizations) to resultant dynamics (Hanski 1998). Initial emphases of metapopulation investigations were on model development, whereas the last two decades have featured the advance of inference methods for fitting such models to empirical data from natural systems. Such data may include sampling of marked animals at multiple locations within a system (Brownie et al. 1993, Lebreton et al. 2009) or species detections and nondetections across multiple locations (MacKenzie et al. 2003, 2006, 2009). An important component of such modeling is the direct incorporation of detection probability parameters that explicitly deal with the ecological reality that our observation of individuals is imperfect. These inference methods permit tests of various concepts that permeate metapopulation theory and, more specifically, of important assumptions incorporated in metapopulation models.

Many data-driven inferences about metapopulation dynamics have been based on the general incidence function approach developed by Hanski (1992, 1994), in which the fraction of patches observed to be occupied is used to estimate extinction and colonization probabilities under the assumption that these parameters are constant over time and that the probability (incidence) of patch occupancy is at long-term equilibrium (Hanski 1994, Moilanen 1999, Clinchy et al. 2002). This approach to inference requires knowledge of functional relationships between patch characteristics and probabilities of local extinction and colonization. Thus, extinction probability has traditionally been modeled as a function of patch size, and colonization probability has been assumed to be a function of patch connectivity with other local populations (Moilanen and Nieminen 2002). More recently, however, investigators have focused on addressing many of the assumptions of metapopulation theory, testing these 
assumptions with empirical data and developing more sophisticated model parameterizations that incorporate biological detail needed to explain observed patterns in patch turnover processes. Such efforts have included integration of dynamic disturbance and habitat succession models to relax traditional assumptions of a static landscape (Ellner and Fussmann 2003, Wilcox et al. 2006, Vuilleumier et al. 2007, MacKenzie et al. 2011, Miller et al. 2012). Other studies have considered the effects of stage structure and synchronization of dynamics across patches on extinction and colonization processes (Sutherland et al. 2012), within-patch dynamics (Crone et al. 2001, Sanderlin et al. 2012) or individual behavior on metapopulation performance (Winfree et al. 2005). The role of matrix structure and configuration on patch extinction/ colonization has been evaluated (Cook et al. 2004, Bender and Fahrig 2005, Kennedy et al. 2011), and simulations or empirical data have been used to test common assumptions relating landscape indices of patch size, isolation, and habitat structure to connectivity and dispersal in order to predict metapopulation occupancy and turnover (Crone et al. 2001, Moilanen and Nieminen 2002, Bender and Fahrig 2005, Winfree et al. 2005, Pellet et al. 2007, Prugh et al. 2008, Kennedy et al. 2011). Lacking in many metapopulation models, however, is an explicit treatment of imperfect detection (but see Pellet et al. 2007, Kennedy et al. 2011).

The metapopulation concept has largely replaced island biogeographic thinking as a framework for conservation decisions (Hanski and Simberloff 1997). Vulnerability of rare and endangered species to extinction has frequently been linked to habitat loss and fragmentation that influence the spatial extent and configuration of existing populations (Caughley 1994, Nicholson and Ovaskainen 2009). In the context of the declining population paradigm (Caughley 1994), space and location are important, as reductions in area occupied by the species may result in fewer and more isolated sources of colonizing individuals. Invasive species, a different sort of conservation problem, are similarly modeled using metapopulation concepts with a focus on local probabilities of extinction and colonization that may themselves be influenced by the occupancy status of neighboring locations (Bled et al. 2011, Yackulic et al. 2012).

One focus of this paper is on the incorporation of additional realism into occupancy models, building on the advances described above as well as recent work of Royle and Dorazio (2008), Bled et al. (2011) and Yackulic et al. (2012). We pose several common assumptions of metapopulation theory (including patch size - extinction and neighbor occupancy - colonization relationships) as hypotheses to be tested. We use multi-season occupancy models (MacKenzie et al. 2006, 2009) which we view as spatially realistic in that they provide inference on local probabilities of patch extinction and colonization as a function of patch characteristics such as size, distance from source population and additional spatial measures of connectivity with other system patches. Such models use patch detectionnondetection data that are collected over time and that allow for nondetection at some occupied patches. Specifically, we use an approach that models patch-level vital rates (colonization and extinction) as functions of focal patch characteristics as well as the occupancy status of neighboring patches. Importantly, this model defines 'neighborhood' as a very general concept, such that it is capable of incorporating biological detail on animal movement, matrix structure, land-cover types, etc. that are believed to be important predictors of species response and patch dynamics (Bender and Fahrig 2005, Winfree et al. 2005, Kennedy et al. 2011). The added difficulty of modeling neighborhood occupancy rather than using neighboring patch characteristics as a proxy for occupancy is that this value cannot be treated as a known covariate such as patch size, cover type or distance. The occupancy of neighbor patches must instead be estimated while accounting for the reality of possible nondetection. Our approach differs from that of most other attempts to incorporate the influence of a neighborhood effect (e.g. through measures of connectivity; Crooks et al. 2001, Robles and Ciudad 2012) by focusing on the actual occupancy status of neighboring patches rather than simply on a metric reflecting connectivity to potential source locations (but see Moilanen and Nieminen 2002, Winfree et al. 2005). We include the explicit treatment of neighbor patch occupancy as a latent state, the dynamics of which are modeled in the same manner as for the focal patch.

Our motivation for this study is a metapopulation of the endangered Lower Keys marsh rabbit Sylvilagus palustris hefneri (LKMR), a species for which habitat loss and fragmentation, and resulting neighborhood effects, are thought to be important determinants of population persistence. LKMR are not readily observable, leading to the use of sign surveys in conjunction with occupancy modeling methods that explicitly deal with nondetection (Eaton et al. 2011, Schmidt et al. 2011b). The LKMR Recovery Plan (US Fish and Wildlife Service 1999) specifies presence/ absence monitoring (including in areas believed to be unoccupied) to determine marsh rabbit distribution and site occupancy as a means for evaluating species status. Historical pellet surveys of LKMR initiated in the 1970s focused on occupancy of patches but did not include replicated, within-season surveys usually required to estimate detection probability. Beginning in 2009, the survey protocol was modified to explicitly address the issue of nondetection through the use of spatially replicated samples within habitat patches (Eaton et al. 2011). Rather than discarding historical data, here we develop a likelihood approach that uses data from surveys with and without geographic replication in an attempt to estimate quantities (e.g. patch-specific probabilities of extinction and colonization) governing occupancy dynamics. Given the constraints imposed by this data structure, we did not think it would be possible to model both detection and extinction/colonization probabilities as fully time-varying (Fujiwara and Caswell 2002). Recognizing that the parameters governing patch turnover may be influenced by temporal processes (e.g. periodic storm events or gradual sea-level rise) we instead included models to test 1) temporal trends in extinction and colonization rates and 2) year-specific changes in vital rates associated with significant high tide events. Temporal variation in extinction and colonization of specific patches could also arise through variation in neighborhood occupancy. The modeling approach explicitly incorporates detection 
probability parameters as well as autologistic parameters that permit inference about the relevance of neighborhood occupancy to extinction and colonization for a focal patch.

Thus, a second focus of this paper is to test several hypotheses (articulated below) about changes in occupancy, with patch location, habitat, and neighborhood occupancy as potential determinants of local probabilities of extinction and colonization. These objectives required the ability to model historical data collected without replicate samples for most years of the investigation. A proximate objective was therefore to develop and assess a model that could make use of both modern and historical data.

\section{Methods}

\section{Occupancy modeling}

Our modeling was based on the multi-season occupancy models of MacKenzie et al. (2003), using detectionnondetection data for a number of sites collected at multiple primary sampling occasions over which occupancy may change. Occupancy dynamics for a focal patch $i$ are modeled as a first-order Markov process in which the probability of occupancy in year $t+1$ is conditional on the occupancy state in the current year, $t$, such that

$\psi_{i, t+1}=\gamma_{i, t}\left(1-\psi_{i, t}\right)+\left(1-\epsilon_{i, t}\right) \psi_{i, t}$

where $\psi_{i, t}$ is the probability of occupancy, $\gamma_{i, t}$ is the probability that a currently empty site will become occupied (i.e. via colonization) and $\epsilon_{i, t}$ is the probability that an occupied site will become unoccupied (i.e. local extinction; the complement of local persistence, $\left.1-\phi_{i, t}\right)$ in the following year. In Eq. 1, both extinction and colonization probabilities are described in their most general form as time-specific, but they can also be modeled as constant.

\section{Autologistic modeling}

Multi-season occupancy models (MacKenzie et al. 2003, 2006, 2009) are very flexible and permit modeling of local probabilities of extinction and colonization as functions of patch characteristics (e.g. habitat) and time-specific (e.g. climate, management) covariates. However, these basic models have not allowed the incorporation of neighborhood effects in which vital rates of a focal patch are affected by the occupancy status of neighbors, unless neighbor status is known with certainty. Such neighborhood effects are widely considered in metapopulation theory (e.g. in the form of connectivity or proportion of overall patch occupancy of the metapopulation) and provide another important ecological mechanism that can be incorporated into occupancy modeling. Following the recent work of Royle and Dorazio (2008), Bled et al. (2011) and Yackulic et al. (2012), we developed an autologistic neighborhood model for our study system.

The occupancy status of a neighboring patch, $j$, may not be known with certainty, just as the status of a focal patch, $i$, is often not known with certainty (i.e. because it was not surveyed in a given year or because it was surveyed but no individuals were detected). Unlike standard, site-specific covariates that are measured directly, the autologistic covariate is treated as a latent variable and must be estimated. For any focal patch, some or all of its neighbor patches may be surveyed in the course of a given sampling occasion (e.g. year). We assume that the study organism can be identified, when present, without error (no false positives); thus, when surveys yield a detection, the neighbor patch is considered as occupied with no uncertainty. When surveys yield no detection, the conditional (on no detection) probability of occupancy can be computed (MacKenzie et al. 2006: 97-98) and used in the autologistic modeling. When a neighbor patch is not surveyed, the unconditional probability of occupancy can be used in the autologistic modeling. Thus, as long as neighbor patches can be identified and patchspecific covariates, if any, can be measured, autologistic modeling can be carried out using all potential neighbor patches, regardless of whether they are surveyed.

Considering the characteristics and biology of the species under study, one may define the relevant 'neighborhood' in any number of ways. Commonly, such a definition will be based on some distance, $d$, separating the focal patch and potential neighbor patches. Conditional on a specified neighborhood, the covariate used in autologistic modeling is then the product of 2 quantities. The first quantity is the weighted average occupancy $\left(\widehat{\bar{\psi}}_{i, t}^{N_{i}}\right)$ for the set of neighborhood patches $\left(N_{i}\right)$ that are potential sources of colonists for focal patch $i$ at time $t$. This average is based on patch-specific weights $\left(w_{i j}\right)$ that can be computed in a variety of ways depending on the patch characteristics most likely to influence the number of potential colonists that reach the focal patch. Whereas some metapopulation models have treated neighborhood patches as binary (i.e. suitable habitat vs inhospitable matrix), our approach is to permit neighbor patches to vary in their potential for contributing immigrants to a focal patch, based on any number of biological hypotheses relevant to the focal species. Such factors could include the size of each neighbor patch in $N_{i}$, patch habitat quality, or measures of habitat connectivity or resistance between the focal patch and each neighboring patch. The latter quantities fall under the general concept of modeling cost-weighted 'effective distances' between nodes as a function of landscape characteristics hypothesized to impede or facilitate movement, and includes least-cost path (Epps et al. 2007, Joshi et al. 2011, Royle et al. 2013) and graph theory methods (McRae et al. 2008, Lookingbill et al. 2010). The second quantity, $\eta_{i}$, can be viewed as the proportion of the neighborhood area of focal patch $i$ that contains patches of any quality capable of producing colonists. This quantity is intended to address the possibility that some portion of the neighborhood may be comprised of non-habitat (i.e. area with no possibility of producing colonists). Additional detail and the specification of both components of the autologistic covariate, $\eta_{i} \widehat{\bar{\Psi}}_{i, t}^{N_{i}}$, are provided in the Supplementary material Appendix 1.

We note that this flexible approach for defining a neighborhood is conditional on the selected distance, $d$, used to identify potential neighbor patches. Instead of defining the neighborhood extent, some metapopulation models measure the distances between the focal patch and all 
non-focal patches, $d_{i, j}$, to estimate a scaling parameter that defines the probability of moving between patches (i.e. a dispersal kernel; Sutherland et al. 2012). Our model allows the distance of neighboring patches within the buffer to be incorporated by means of the weight, $w_{i j}$, permitting researchers the flexibility of incorporating a range of biological hypotheses related to dispersal distance when estimating average neighborhood occupancy and its influence on the dynamics of a focal patch. Selecting a buffer distance to delineate the effective neighborhood is also a practical consideration when the study system is deemed to be contiguous and where including all inter-patch distances (i.e. based on the centroid of each cell in a grid design) would be computationally costly. Choice of $d$ should ideally be based on knowledge of movement and dispersal capabilities of the focal species. However, there will frequently be uncertainty about the most appropriate value for $d$. In such cases, a reasonable approach involves multiple models in which neighborhoods are defined by different values of $d$ that encompass the range of potential distances. Model selection or likelihood ratio testing could then be used to identify the value of $d$ that is most consistent with the data, thus providing inference on the effective neighborhood size. This is the approach we take below.

\section{Covariates}

In addition to these neighborhood effects, we wanted to model probabilities of local extinction and colonization as functions of habitat characteristics of the focal patch itself. For example, if $X_{i}$ is the value of a habitat covariate for patch $i$, and $\eta_{i} \widehat{\bar{\psi}}_{i, t}^{N_{i}}$ is the autologistic covariate for this patch as defined above, then we can model vital rates for focal patch $i$ at time $t$ as follows:

$\operatorname{logit}\left(\theta_{i, t}\right)=\beta_{0}+\beta_{1} X_{i}+\beta_{2, d} \eta_{i} \widehat{\hat{\psi}}_{i, t}^{N_{i}}$

where $\theta_{i, t}$ is the parameter to be estimated (annual probability of extinction or colonization for patch $i$ ), $\beta_{0}$ is an intercept term, $\beta_{1}$ is the coefficient for the patch-specific habitat covariate, and $\beta_{2, d}$ is the coefficient for the timeand patch-specific neighborhood covariate at the effective neighborhood size $d$.

\section{Lower Keys marsh rabbit}

We applied models that incorporated these effects to detection histories collected over a period of 22 years (1991-2012) for populations of the Lower Keys marsh rabbit Sylvilagus palustris hefneri, an endangered sub-species endemic to the lower Florida Keys. Although federally protected, populations of this historically widespread and abundant lagomorph have continued to decline due to habitat loss and degradation, predation by cats, vehicle collisions, fire suppression and storm events (US Fish and Wildlife Service 1990, 1999, 2007). Because LKMR metapopulations are restricted to small, fragmented patches with limited connectivity (Forys 1995, Faulhaber et al. 2007, US Fish and Wildlife Service 2007), the use of occupancy models is appropriate for estimating probabilities of local patch extinction and colonization as a function of intrinsic patch characteristics and the occupancy status of neighboring patches while accounting for issues of detectability. Below, we summarize the salient points of modeling the dynamics of LKMR using multi-season occupancy with an autologistic covariate. For interested readers, we provide additional details on the study area, survey methods and ecology of this species in the Supplementary material Appendix 2.

\section{Study area}

Florida's Lower Keys are comprised of a linear array of large keys (mainline keys) surrounded by smaller keys (outer keys), each of which varies in size, shape, elevation and orientation to its neighbors (Fig. 1). The range of the LKMR extends from Big Pine Key and its outer islands in the east (hereafter 'BPK'), to Sugarloaf, Saddlebunch, and Boca Chica and associated outer keys in the west (hereafter 'SBC'). The two metapopulations of LKMR found on SBC and BPK have been described as genetically and geographically distinct clades ('western' and 'eastern', respectively), and are found on either side of a set of islands containing suitable habitat patches but believed to have been extirpated of LKMR in recent decades (we refer to this 'gap island complex' as GAP; Howe 1988, Lazell 1989, Faulhaber et al. 2007, Crouse et al. 2009, Tursi et al. 2012) (Fig. 1). Documented and potential LKMR habitat patches were catalogued by Faulhaber (2003) and Faulhaber et al. (2007) across 29 islands in the lower Florida Keys. This set of legacy patches, which comprise all previously known LKMR habitat localities ( $\mathrm{n}=228$ patches), was used for our analysis. None of the patches included in this study is thought to have been lost to sea-level rise or become uninhabitable due to habitat disturbance, despite claims of significant habitat loss in this system (Schmidt et al. 2012).

\section{Survey methods and detection probability}

Surveys for LKMR populations have been conducted on a near-annual basis in the Lower Keys beginning in the late 1980s (Howe 1988). Although a variety of LKMR research and monitoring programs have taken place subsequently in the Lower Keys, leading to methodological differences in survey design and data collection, most monitoring efforts have relied on fecal pellet surveys to detect the presence of LKMR (Howe 1988, Forys 1995, Forys and Humphrey 1996, Faulhaber et al. 2007, Schmidt 2009, Schmidt et al. 2011a). Prior to 2009, most pellet count surveys were not designed to account for issues of detectability (i.e. the probability of detecting the presence of at least one rabbit, or associated sign, in a patch, given that the patch is occupied). Beginning in 2009, we redesigned the pellet survey protocol in order to draw separate inferences about detection probability and the parameters that describe patch occupancy dynamics (for more detail on the survey design, see Supplementary material Appendix 2 and Eaton et al. 2011). Briefly, our survey design involved placing $12 \mathrm{~m}$-diameter circular plots, randomly and with replacement, in LKMR habitat patches which were surveyed without time constraints. We include in our analysis 


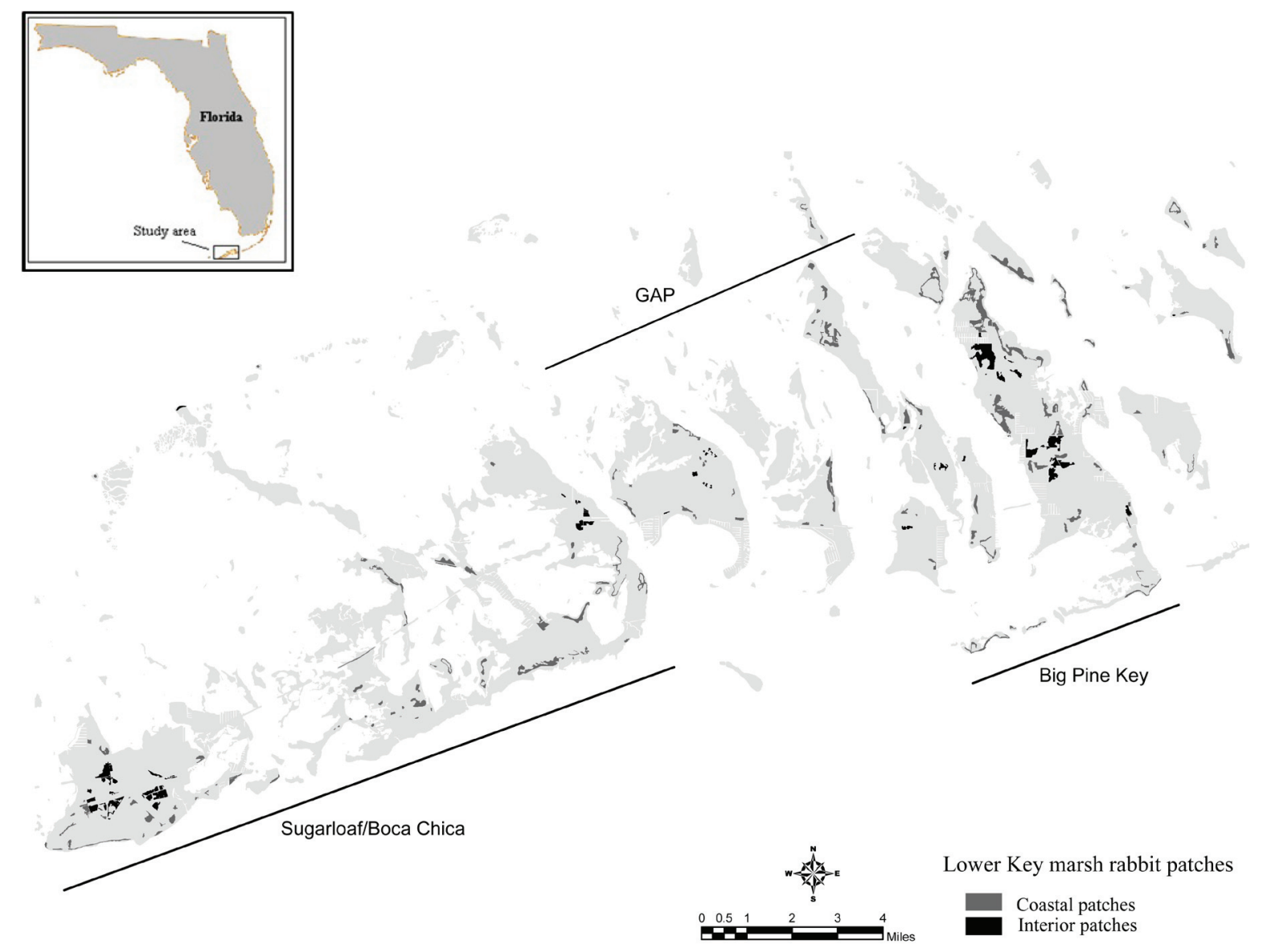

Figure 1. Map of the Lower Florida Keys, USA. Patches represent all known extent of Lower Keys marsh rabbit habitat; variation in shading indicates designation as coastal (grey) or interior (black) patches. Purported metapopulations (Sugarloaf-Boca Chica, Big Pine Key and Gap Islands) are denoted.

detection histories from surveys conducted prior to 2009 (hereafter, referred to as 'historical' data), augmented with data from four years (2009-2012) of surveys using the revised protocol (termed the 'modern' data set). Because of a number of gaps in survey years and uncertainty in survey methods prior to 1991 , the historical data set was restricted to the years 1991-2008. We grouped historical survey years by similarity of study method (Supplementary material Appendix 4 Table A4.3) in order to test whether vital rates and detection probabilities could reliably be estimated over this period by including the modern survey histories in the analysis and imposing constraints on other model parameters (Fujiwara and Caswell 2002, Kendall and Nichols 2002). Specifically, we constrained extinction and colonization probabilities to vary over time via changes in annual neighborhood occupancy (neighbor effect) or by means of a restricted parameterization (i.e. a simple linear trend or binary indicator of storm years requiring few additional parameters).

We compared models that assumed detection probability, $p$, was constant across the study period, that allowed $p$ to vary annually, and that constrained $p$ as constant for a given survey method but variable among methods. We also tested variability in detection as a function of patch size and location with respect to the coastline to account for potential additive effects of search method and patch covariates in the historic surveys.

\section{LKMR patch covariates}

We believe that extrinsic factors related to the geology and topography of the western, gap and eastern islands may have resulted in distinct patch occupancy dynamics among these three metapopulations (Forys 1995, US Fish and Wildlife Service 2007) and, therefore, designated each patch according to its metapopulation membership (BPK, SBC or GAP) by means of a dummy variable. We refer to models that include separate parameters designating metapopulation membership collectively as ' $P O P$ '.

Additional patch covariates identified from a priori hypotheses of factors likely to influence LKMR dynamics included patch size $(\mathrm{Ha})$ and the location of a patch with respect to coastline (location). The rationale for the covariate $H a$ was provided elsewhere (Eaton et al. 2011) and is consistent with basic metapopulation theory. The covariate location is a binary designation (coastal: location $=0$; interior: location $=1$ ) used to differentiate between major habitat types associated with the presence or absence of both tidal influence and susceptibility to tropical storms. 
To test the autologistic covariate against the survey data, we proposed several hypotheses related to the dispersal abilities of LKMR. These were expressed in the form of varying patch buffer distance classes $(d)$ used to define the boundary strip, and hence the neighborhood extent, for each focal patch. Based on dispersal events observed in other studies (Forys and Humphrey 1999, Faulhaber et al. 2006),we hypothesized that neighborhoods of $500 \mathrm{~m}$ or $1000 \mathrm{~m}$ might be most appropriate for this system, but we considered neighborhood distance classes of 100, 250, 500, 1000, 1500 and 2000 meters. We compared models with these various distance classes using an AIC model selection framework in order to infer the effective neighborhood size of the LKMR.

\section{Temporal covariates}

To evaluate the effect of sea-level rise or tropical storm events on colonization/extinction probabilities, we tested for variation in these vital rates using yearly site covariates. Recognizing that the existing data structure limited our ability to model both ecological and observation processes as fully time-dependent, we minimized additional parameters by 1) modeling extinction and colonization as functions of year and location to identify linear trends in vital rates for both coastal and interior patches, 2) assigning a binary indicator for years in which tropical storms reaching the Lower Keys may have affected extinction and colonization processes on coastal and interior patches, and 3) testing for changes in vital rates over two periods (1991-2000 and 2001-2012) and between patch locations. We identified 1992, 1995, 1999, 2007-2010 and 2012 as years with a maximum average monthly mean high water level (MHW) greater than or equal to $0.4 \mathrm{~m}$ above mean sea level, based on observations of severe tidal influx for Monroe County, FL (NOAA Hurricane Research Division; $<$ www.aoml.noaa.gov/hrd/hurdat/Data_Storm.html $>$ ).

\section{Parameterization}

We modeled annual probabilities of patch extinction and colonization as functions of the covariates discussed above and the basic framework of Eq. 2. We used a common parameterization of the multi-season occupancy model in which the probability of occupancy is estimated for the initial sample period $\left(\psi_{i, t}\right.$; for $\left.t=1\right)$ along with annual extinction $\left(\epsilon_{i, t}\right)$ and colonization $\left(\gamma_{i, t}\right)$ probabilities for all intervals between sample periods (MacKenzie et al. 2006, p. 187-198). The most general model for a vital-rate parameter for our study system is then

$$
\begin{aligned}
\operatorname{logit}\left(\theta_{i, t}\right)= & \beta_{1} S B C_{i}+\beta_{2} B P K_{i}+\beta_{3} G A P_{i}+\beta_{4}\left(\frac{H a_{i}-\overline{H a}}{S D_{H a}}\right)+\beta_{5} \text { location }_{i} \\
& +\beta_{6, d} \eta_{i} \hat{\bar{\Psi}}_{i, t}^{N_{i}}+\beta_{7} \text { year }+\beta_{8}(\text { year } \times \text { location })
\end{aligned}
$$

where $\theta_{i, t}$ is the parameter of interest. Focal patch size $\left(H a_{i}\right)$ has been centered on zero and scaled by one standard deviation. In addition to the annual influence of the neighborhood effect, the covariate year represents one of three alternative ways we include a temporal component in the parameter estimation (see Temporal covariates). When testing for a linear trend, year was centered on zero by subtracting the mean year [2001.5] for each survey year. Each of these year-dependent covariates is also modeled as an interaction with location to accommodate differential extinction and colonization rates between coastal and interior sites. Note that the coefficient used for the autologistic covariate $\left(\beta_{6, d}\right)$ is specific to the buffer distance class (d) being assessed. Because we did not have a priori hypotheses regarding variation in the quality of potential neighbor patches as contributors of colonizing rabbits, we assumed that all neighbors of a focal patch were of equal value (i.e. all $w_{i j}=1$ ).

Due to the large number of possible parameter combinations to consider for a model set, our model selection approach was to divide the analysis into subsets. We emphasize that this stepwise approach to model selection is not the only way to deal with a large number of potential models, but it was seen as a reasonable and practical way to proceed. Models were fit using a modified version of the program Presence (Hines 2006). We report linearlogistic $\beta$ coefficient estimates for each well-supported model (i.e. $\triangle \mathrm{AIC} \leq 2.0$ ) and present model-averaged estimates of real parameters when useful for inference on metapopulation dynamics of LKMR. Further details and the model selection results are provided in the Supplementary material Appendix 4.

\section{A priori hypotheses for model predictions}

Two of our predictions come directly from basic metapopulation theory: patch extinction probability should be a decreasing function of patch size, and patch colonization probability should be an increasing function of neighborhood occupancy. Because larger patches should support a greater abundance of individuals, and thus reduce the probability of local extinction resulting from demographic processes, we anticipated that $\beta_{4}$ (patch size, from Eq. 3) would be negative for extinction probability. We hypothesized that estimates for $\beta_{4}$ and $\beta_{6}$ (autologistic covariate) would be positive for colonization, reflecting the belief that dispersing individuals would have a greater probability of encountering larger patches and that higher average neighborhood occupancy would increase the likelihood of colonization in focal patches, respectively. Additionally, we predicted a negative value for $\beta_{6}$ of the extinction model based on the rescue effect (Brown and Kodric-Brown 1977), which hypothesizes a reduced extinction rate when prospective colonizers in a patch's neighborhood can offset rates of local extirpation. Based on historical, largely anecdotal information regarding the relative stability of the three metapopulations we hypothesized for both initial occupancy and colonization that $\beta_{1}>\beta_{2} \gg \beta_{3}$, and that estimates of extinction probability coefficients would be $\beta_{1}<\beta_{2} \ll \beta_{3}$ (where the $\beta$ s are intercept coefficients for SBC, BPK and GAP islands, respectively). Because sea-level rise and amplified impacts of storm events are resulting in gradual changes in the Florida Keys (Ross et al. 2009), we hypothesized that these dynamics would result in changes to colonization and extinction rates over this 22-year period. Considering possible time-dependent effects of storm surge 
and sea-level rise, we anticipated reduced colonization and increased extinction over time. However, we hypothesized that the magnitude of such effects would be more pronounced on coastal than on interior patches. We discuss additional a priori model predictions in the Supplementary material Appendix 2.

\section{Simulation analysis}

We desired a model that incorporated detection probabilities, but were uncertain that our modeling approach would 'work', i.e. that all parameters were identifiable and could be estimated with our data. We were confident that the modern, replicated surveys conducted since 2009 provided adequate data for modeling, but we had doubts about the 'historical' years for which there was only a single observation per patch per year. The analogy of multiseason models of occupancy dynamics with temporary emigration capture-recapture models led us to believe that some modeling could be possible with such singleobservation data (Fujiwara and Caswell 2002, Kendall and Nichols 2002, Dail and Madsen 2011). In addition, the work of Lele et al. (2012) shows the possibility of inference in single-season models without replicate visits, as long as certain conditions on covariate relationships are met. Our approach was to first fit a number of models to these data and, if convergence was attained with these models, to use model selection to determine which hypothesis was best supported by the data. Rather than investigate properties of very general models for which we strongly suspected identifiability problems, we then based a simulation study on the actual data analyses, focusing on the constrained models that appeared to be most informative for these data. To evaluate model performance, we quantified the bias and percent relative bias for vital rate parameters across a range of covariate values. To confirm that the model was performing as expected, we increased the number of patches 10 -fold (maintaining the proportional distribution of all covariate values) and simulated the observation data under complete sampling (i.e. no missing data). Additional details are provided in Supplementary material Appendix 3.

\section{Results}

\section{Model selection results}

Model selection (detailed results provided in Supplementary material Appendix 4) supported two models that differed only in their fitting of the data to the detection process. In both cases, the observed detection histories supported the following ecological model (Supplementary material Appendix 4 Table A4.4):

$$
\begin{aligned}
& \psi_{1}(P O P), \gamma\left(P O P+\text { location }+H a+\eta_{i} \hat{\bar{\psi}}_{i, 1000, t}^{N_{i}}+\text { year }+ \text { year } \times \text { location }\right) \\
& \epsilon\left(P O P+\text { location }+H a+\eta_{i} \hat{\bar{\Psi}}_{i, 1000, t}^{N_{i}}+\text { year }+ \text { year } \times \text { location }\right)
\end{aligned}
$$

where the covariate year represents an annual trend. Subsequent parameter estimates, including model-averaged estimates, simulations and inferences about individual patch dynamics, were based on fitting this model for the vital rates. Even with the inclusion of time-dependent parameters for both vital rates and detection probability, the models demonstrated numerical convergence. The AIC bestsupported model included annual variation in detection probability $[p(t)$, with model weight (see MacKenzie et al. 2006, hereafter: $\left.w t_{i}\right)=0.61$ ], while a model of detection probability that varied by both year and location $[p(t+l o c)]$ received some support $\left(w t_{i}=0.39\right.$, Supplementary material Appendix 4 Table A4.4).

\section{Parameter estimates and occupancy dynamics}

Estimates of $\beta$ coefficients for the two highest-ranking models were generally consistent with predictions regarding direction and magnitude, but not in all cases (Table 1). Our central hypotheses regarding the influences of both patch size and neighborhood effect on colonization and extinction were supported, which included an effective neighborhood size of $d=1000$ meters from a focal patch's border (Supplementary material Appendix 4 Table A4.2, A4.3). Estimated coefficients for patch size and neighborhood occupancy were positive for colonization probabilities and negative for extinction probabilities (Table 1), corresponding to predictions of metapopulation theory. Relatively large $\beta$ estimates for the autologistic covariate reflect the highly fragmented nature of our study system - the proportion of rabbit habitat in the neighborhood $(d=1000 \mathrm{~m})$ of a given patch ranged from zero to $0.2(\bar{x}=0.04)$. The neighborhood effect appeared to have a significant influence on colonization probability such that even small patches experienced high rates of colonization when located near other occupied habitat, particularly in coastal areas (Fig. 2).

Our hypotheses for coefficients associated with the three island metapopulations were supported by the data. Under both top-ranked models, the signs and, generally, magnitudes of individual coefficients were the same (Table 1). Results suggest that interior sites were colonized at lower rates than coastal sites, but that inland habitat may have greater stability and serve as refugia to LKMR by providing reduced extinction rates relative to those of coastal patches (Fig. 3, additional details provided in Supplementary material Appendix 5).

Model selection supported two sources of temporal variation in extinction and colonization probabilities: 1) changes in annual neighbor occupancy and, 2) a year effect modeled as a monotonic trend which differed for interior and coastal sites. A negative interaction between year and location (Table 1) suggests that colonization has increased over time, but more slowly at interior sites relative to the coast. Models that excluded an annual trend for colonization received virtually no support (Supplementary material Appendix 4 Table A4.1). Extinction probability was also estimated to have increased over time, but at a faster rate for coastal relative to interior patches (Table 1). Using Eq. 1 and the structure of the topranked models, we applied model-averaged estimates of initial occupancy and patch-specific probabilities of colonization and extinction to estimate occupancy dynamics for all patches during the 22 study period (Fig. 4, GAP keys not shown). Considering only the past ten years (2003-2012), SBC was estimated to have higher 
Inland

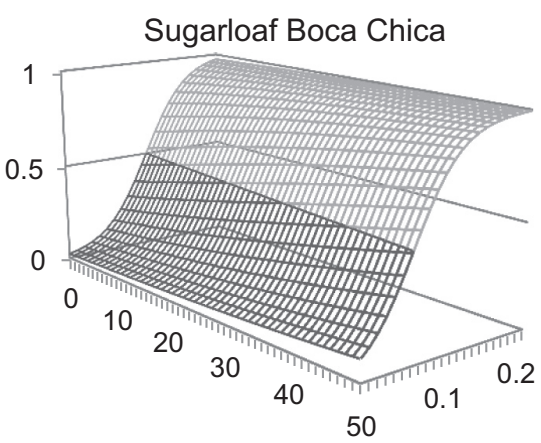

Big Pine

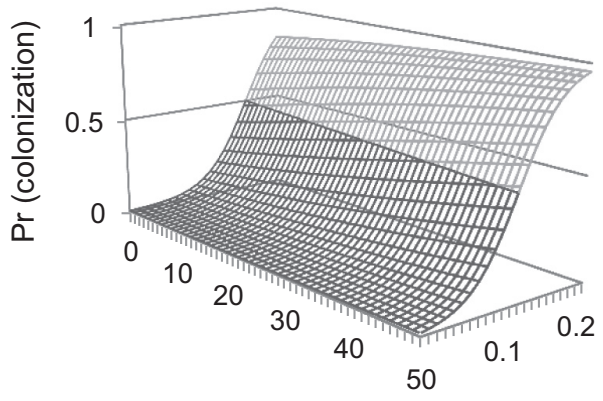

GAP

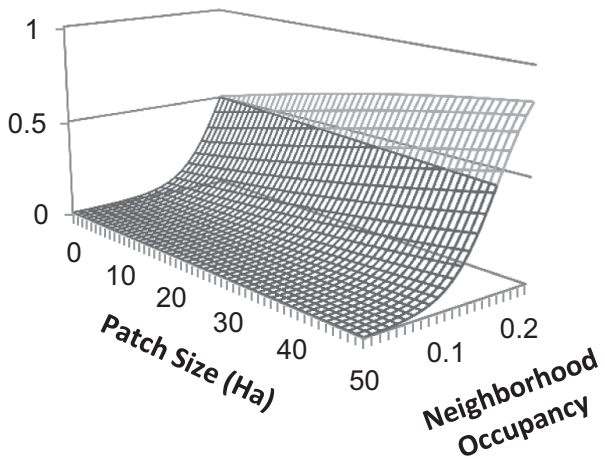

Coastal

Sugarloaf-Boca Chica

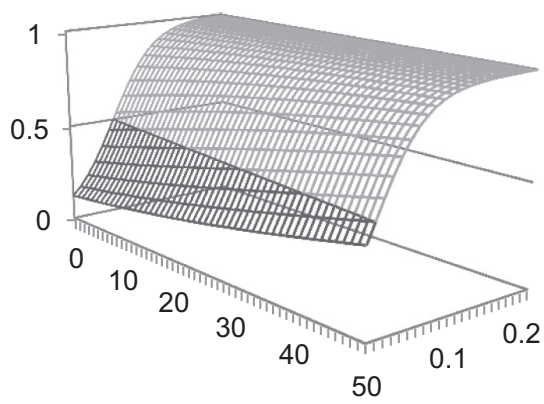

Big Pine

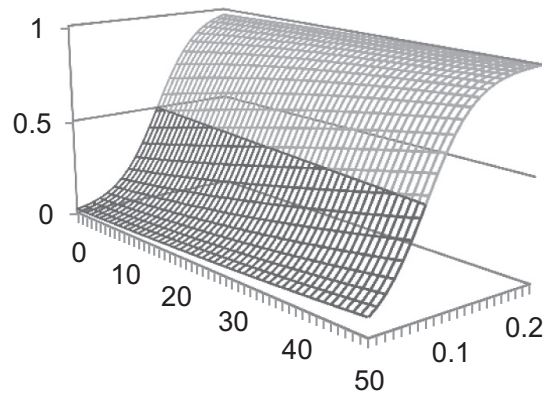

GAP

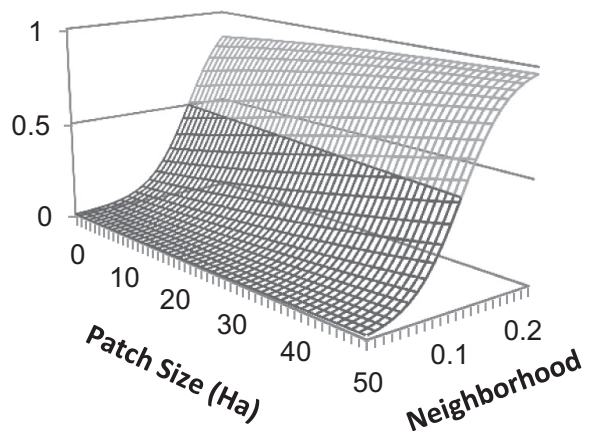

Figure 2. Estimated colonization probability for Lower Keys marsh rabbits as a function of membership in one of three metapopulations, patch size, patch location (interior, coastal) and the autologistic covariate (product of weighted average neighborhood occupancy and the proportion of rabbit habitat within focal patch neighborhood). SBC refers to patches on Sugarloaf, Saddlebunch and Boca Chica Keys.

overall occupancy $\widehat{\bar{\psi}}=0.59, S D(\widehat{\bar{\psi}})=0.21)$ than patches on BPK. $\widehat{\hat{\psi}}=0.45, S D(\hat{\bar{\psi}})=0.34)$. The difference in average annual occupancy between interior and costal sites was also smaller on SBC than on BPK (0.18 vs 0.52 , respectively) over the past 10 years. During this same period, GAP patches had an estimated mean patch occupancy of $<0.01$. Lower extinction rates of interior sites contributed to higher average occupancy than in coastal patches. However, isolated inland patches were estimated to have lower occupancy than the majority of coastal sites, and a purely a posteriori evaluation of the dynamics in Fig. 4 suggested the presence of a possible threshold in the occupancy of inland sites. On further inspection we determined that all interior patches with neighborhoods comprising $<6 \%$ suitable habitat (i.e. $\eta_{i}<0.06$; see Supplementary material
Appendix 1 for parameter description) had much lower estimated occupancy levels $(<0.4)$ over time than patches with $\eta_{i} \geq 0.06$.

\section{Detection probabilities}

Under the two top-ranked AIC models, variation in detection probability was best described by annual estimates of $p\left(w t_{i}=0.61\right)$ despite consistent survey methods across groups of years; there was some evidence $\left(w t_{i}=0.39\right)$ suggesting annual detection was slightly higher for inland than for coastal sites (Table 1, Supplementary material Appendix 4 Table A4.4). Additional details on the results of the detection probability analysis are provided in Supplementary material Appendix 5. 
Inland
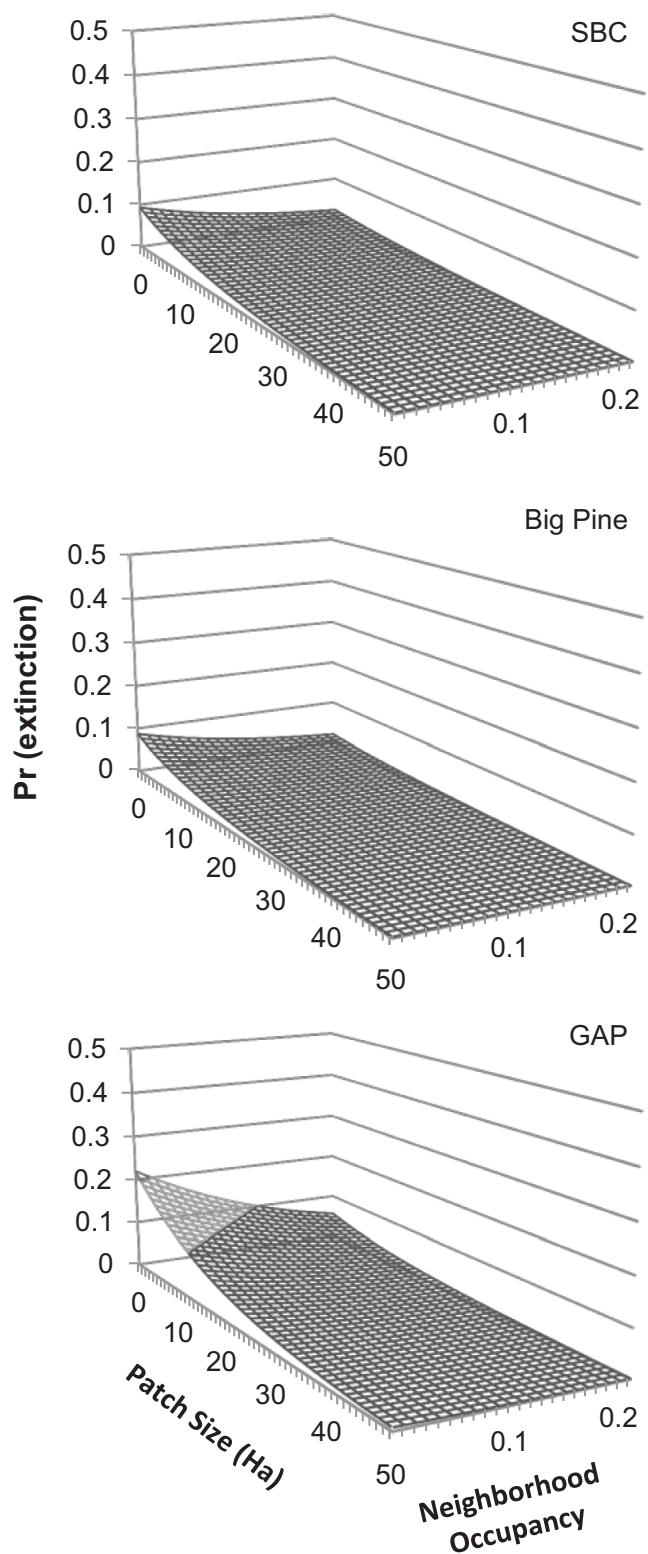

Coastal

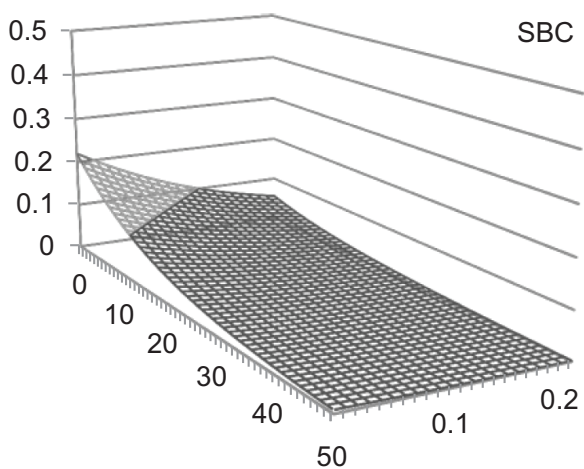

Big Pine
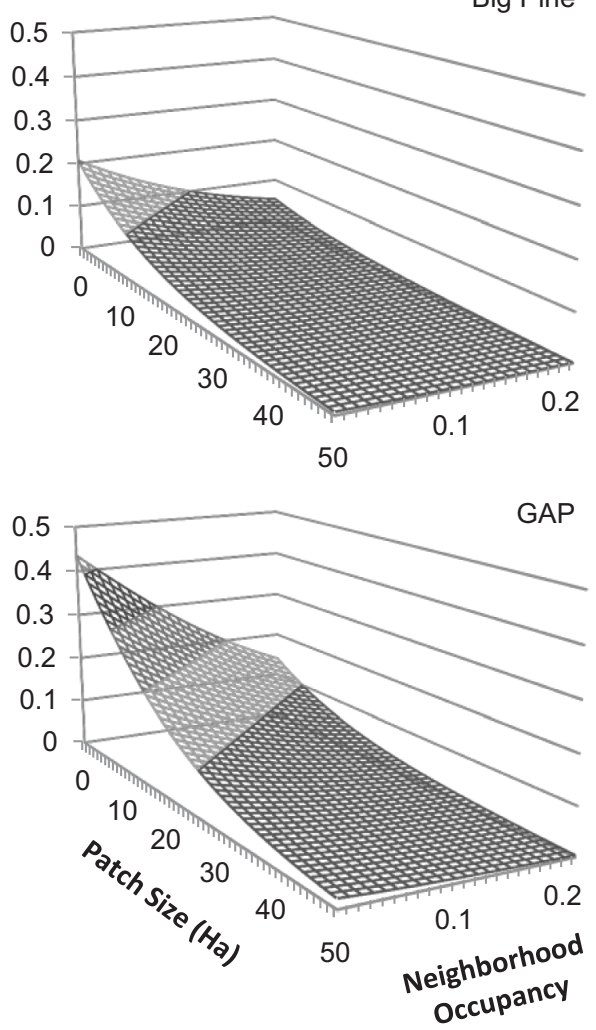

Figure 3. Estimated extinction probability for Lower Keys marsh rabbits as a function of membership in one of three metapopulations, patch size, patch location (interior, coastal) and the autologistic covariate (product of weighted average neighborhood occupancy probability and the proportion of rabbit habitat within focal patch neighborhood). SBC refers to patches on Sugarloaf, Saddlebunch and Boca Chica Keys.

\section{Simulation results}

Simulation results, using the top-ranked model structure and similar coefficient values indicated that the model performed reasonably well. With a 10 -fold increase in the number of simulated patches, an analysis of 50 iterations demonstrated that biases in extinction, colonization and detection were much reduced, with absolute bias in all cases $<0.01$ (data not shown). Additional details regarding simulation results are provided in Supplementary material Appendix 5.

\section{Discussion}

\section{Inferences about LMKR metapopulations}

Our results provide empirical support for two major tenets of metapopulation theory: that colonization is positively related to occupancy of neighboring patches and that local extinction is negatively related to patch size. We also found evidence for two additional relationships that have been incorporated into many metapopulation models: lower extinction rates related to higher neighborhood occupancy 


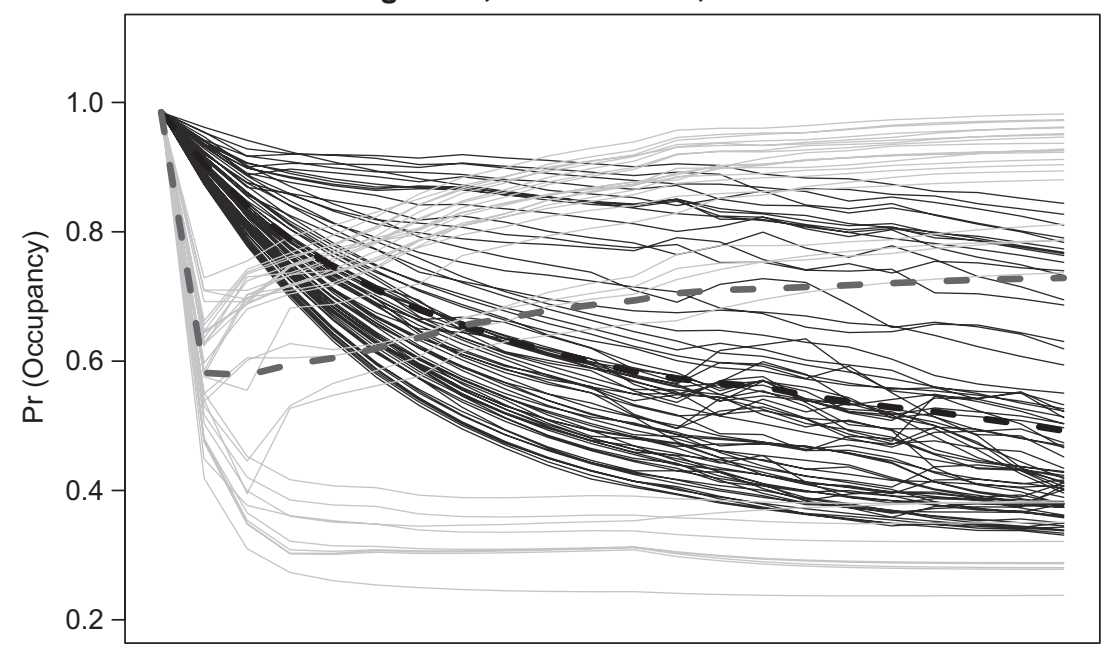

Big Pine

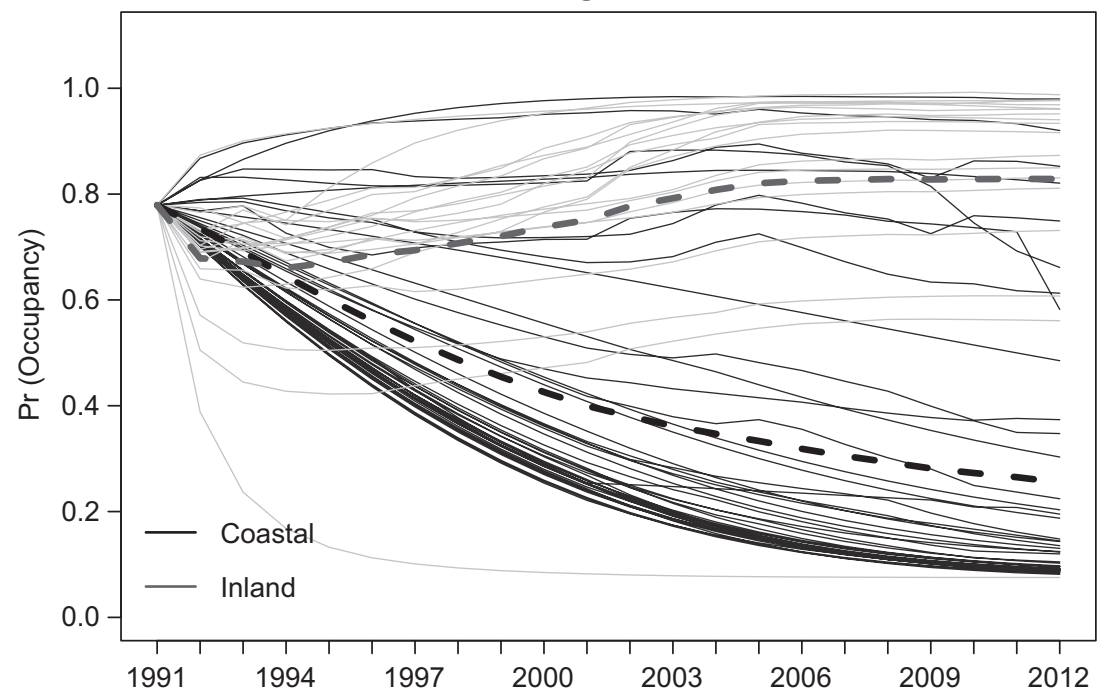

Figure 4. Estimates of patch-specific occupancy $(\psi)$ dynamics for the two primary metapopulations of Lower Keys marsh rabbits during the study period. Beginning with initial occupancy probability, $\psi_{1991}$, patch dynamics were estimated by applying modelaveraged year- and patch-specific probabilities of colonization and extinction to determine the next year's probability of occupancy. Estimated vital rates were derived from the two models best supported by our data set, which suggested that variation in initial occupancy was explained by metapopulation membership; both colonization and extinction were best explained by metapopulation membership, patch location, patch size, a year effect (plus an interaction between year and patch location), and average neighborhood occupancy within $1000 \mathrm{~m}$ of a focal patch. Thin lines represent individual patches, with light gray lines indicating interior and dark grey lines indicating coastal patches. Thick dashed lines represent average patch occupancy by location and metapopulation membership. An observed gap in the estimated occupancy of inland patches may be attributable to a threshold for the proportion of available rabbit habitat within the neighborhood of a focal patch ( $\eta$; see Supplementary material Appendix 1 for a description). All inland patches with $<0.06$ habitat in the $1000 \mathrm{~m}$ buffer were estimated to have considerably lower occupancy probabilities than those with $\geq 0.06$ available habitat.

(rescue effect) and higher colonization rates with larger patch size. The specification of an autologistic covariate allowed us to directly estimate the occupancy status of neighboring patches, accounting for possible nondetection, rather than treat the neighborhood as a proxy for the existence of colonizers. Despite the wide use of such assumptions in metapopulation modeling, empirical evidence based on analyses that include nondetection is surprisingly scarce. Indeed, three recent efforts to investigate these relationships using occupancy modeling were unable to demonstrate support for either hypothesis (Pellet et al.
2007, Kennedy et al. 2011, Yackulic et al. 2014 for northern spotted owls). We are aware of two studies that did provide support for neighborhood effects using autologistic modeling (Bled et al. 2011, Yackulic et al. 2012), but both of these examples were of species in the process invading previously unoccupied areas (i.e. experiencing transient dynamics). As in any regression problem, variation in the covariate must be sufficient to permit estimation of the regression parameters. It is useful to know that spatial variation in neighborhood occupancy across our existing metapopulations (that were not experiencing rapid expansions 
Table 1. Parameter estimates for covariate models from the top two a priori models of occupancy dynamics [both with the structure; $\psi_{1}(P O P), \gamma\left(P O P+\right.$ location $+H a+\eta_{i} \widehat{\widehat{\Psi}}_{i, t}^{N}[1000]+$ Year + Year $\times$ location $), \epsilon\left(P O P+\right.$ location $+H a+\eta_{i} \widehat{\widehat{\Psi}}_{i, 1000, t}^{N}+$ Year + Year $\times$ location $\left.)\right]$

and detection probability [model 1: $p(t)$ and model $2: p(t+l o c)$ ] for Lower Keys marsh rabbits. Where useful, real estimates for vital rates were calculated using covariate combinations. Otherwise, $\hat{\beta}$ s are provided for individual site covariates. Model-averaged estimates and their SEs are also provided. Parameters and covariates are the same as in Supplementary material Table A4.2-4.3 with the covariate POP expanded to its constituent locations [SBC (Sugarloaf, Saddlebunch and Boca Chica), BPK (Big Pine Key) and GAP islands].

\begin{tabular}{|c|c|c|c|c|c|c|c|c|}
\hline \multirow[b]{2}{*}{ Param } & \multirow[b]{2}{*}{ Covariate } & \multicolumn{2}{|c|}{ Model $1(w t=0.614)$} & \multicolumn{3}{|c|}{ Model $2(w t=0.386)$} & \multirow{2}{*}{\multicolumn{2}{|c|}{$\begin{array}{c}\text { Model average } \\
\text { Real (SE) }\end{array}$}} \\
\hline & & $\beta(\mathrm{SE})$ & Real (SE) & $\beta$ (SE) & Real & I (SE) & & \\
\hline \multirow{3}{*}{$\psi_{1}$} & $S B C$ & $4.17(6.11)$ & $0.98(0.09)$ & $231.32(-)$ & & $1.0(-)$ & & $99(-)$ \\
\hline & $B P K$ & $1.26(0.76)$ & $0.78(0.13)$ & $1.27(0.77)$ & & $.78(0.13)$ & & $78(0.13)$ \\
\hline & GAP & $-23.0(-)$ & $<0.001(-)$ & $-122.28(-)$ & $<0.0$ & $01(-)$ & $<0.0$ & $01(-)$ \\
\hline \multirow{8}{*}{$\gamma$} & $\begin{array}{l}\text { location } \\
\quad \text { (interior) }\end{array}$ & $-1.79(0.67)$ & & $-1.8(0.67)$ & & & & \\
\hline & $\mathrm{Ha}$ & $0.19(0.12)$ & & $0.19(0.12)$ & & & & \\
\hline & $S B C$ & $-1.85(0.25)$ & & $-1.85(0.24)$ & & & & \\
\hline & $B P K$ & $-3.83(0.50)$ & & $-3.82(0.50)$ & & & & \\
\hline & GAP & $-5.45(1.11)$ & & $-5.44(1.11)$ & & & & \\
\hline & year & $0.04(0.05)$ & & $0.04(0.05)$ & & & & \\
\hline & year $\times$ loc & $-0.27(0.09)$ & & $-0.28(0.09)$ & & & & \\
\hline & $\eta_{i} \widehat{\bar{\Psi}}_{i, t}^{N}$ & $34.98(7.34)$ & & $35.37(7.42)$ & & & & \\
\hline \multirow{9}{*}{$\varepsilon$} & $\begin{array}{l}\text { location } \\
\quad \text { (interior) }\end{array}$ & $-1.04(0.55)$ & & $-0.97(0.56)$ & & & & \\
\hline & $\mathrm{Ha}$ & $-0.43(0.14)$ & & $-0.43(0.14)$ & & & & \\
\hline & $S B C$ & $-1.52(0.20)$ & & $-1.54(0.2)$ & & & & \\
\hline & $B P K$ & $-1.60(0.24)$ & & $-1.62(0.24)$ & & & & \\
\hline & GAP & $-0.52(1.35)$ & & $-0.52(1.36)$ & & & & \\
\hline & year & $0.07(0.04)$ & & $0.06(0.03)$ & & & & \\
\hline & year $\times$ loc & $-0.34(0.07)$ & & $-0.33(0.07)$ & & & & \\
\hline & $\eta_{i} \widehat{\widehat{\Psi}}_{i, t}^{N}$ & $-7.33(4.99)$ & & $-7.52(5.22)$ & & & & \\
\hline & & $\beta(\mathrm{SE})$ & Real (SE) & $\beta(\mathrm{SE})$ & Real coast (SE) & Real inland (SE) & Real coast (SE) & Real inland (SE) \\
\hline \multirow{19}{*}{$p^{+}$} & 1991 & $1.90(0.65)$ & $0.87(0.07)$ & $1.84(0.62)$ & $0.86(0.07)$ & $0.89(0.06)$ & $0.87(0.07)$ & $0.88(0.07)$ \\
\hline & 1992 & $2.94(1.08)$ & $0.95(0.05)$ & $2.99(1.08)$ & $0.95(0.05)$ & $0.96(0.04)$ & $0.95(0.05)$ & $0.95(0.05)$ \\
\hline & 1993 & $2.73(1.30)$ & $0.94(0.07)$ & $2.77(1.39)$ & $0.94(0.08)$ & $0.95(0.06)$ & $0.94(0.08)$ & $0.94(0.07)$ \\
\hline & 1996 & $1.87(0.82)$ & $0.87(0.10)$ & $1.91(0.87)$ & $0.87(0.1)$ & $0.90(0.09)$ & $0.87(0.1)$ & $0.88(0.09)$ \\
\hline & 1997 & $3.92(5.29)$ & $0.98(0.10)$ & $3.59(3.92)$ & $0.97(0.1)$ & $0.98(0.08)$ & $0.98(0.1)$ & $0.98(0.09)$ \\
\hline & 1999 & $1.30(0.67)$ & $0.79(0.11)$ & $1.22(0.66)$ & $0.77(0.12)$ & $0.81(0.10)$ & $0.78(0.12)$ & $0.80(0.11)$ \\
\hline & 2001 & $24.42(-)$ & $1.0(-)$ & $27.22(-)$ & $1.0(-)$ & $1.0(-)$ & $1.0(-)$ & $1.0(-)$ \\
\hline & 2002 & $27.66(-)$ & $1.0(-)$ & $29.52(-)$ & $1.0(-)$ & $1.0(-)$ & $1.0(-)$ & $1.0(-)$ \\
\hline & 2003 & $4.16(1.83)$ & $0.98(0.03)$ & $4.18(2.2)$ & $0.99(0.03)$ & $0.99(0.03)$ & $0.99(0.03)$ & $0.99(0.03)$ \\
\hline & 2004 & $28.33(-)$ & $1.0(-)$ & $31.16(-)$ & $1.0(-)$ & $1.0(-)$ & $1.0(-)$ & $1.0(-)$ \\
\hline & 2005 & $26.56(-)$ & $1.0(-)$ & $22.97(-)$ & $1.0(-)$ & $1.0(-)$ & $1.0(-)$ & $1.0(-)$ \\
\hline & 2006 & $0.98(0.27)$ & $0.73(0.05)$ & $0.85(0.29)$ & $0.70(0.06)$ & $0.75(0.06)$ & $0.72(0.06)$ & $0.73(0.06)$ \\
\hline & 2007 & $2.03(0.53)$ & $0.88(0.05)$ & $1.86(0.54)$ & $0.87(0.06)$ & $0.89(0.05)$ & $0.88(0.06)$ & $0.89(0.05)$ \\
\hline & 2008 & $1.68(0.38)$ & $0.84(0.05)$ & $1.49(0.4)$ & $0.82(0.06)$ & $0.85(0.05)$ & $0.83(0.06)$ & $0.85(0.05)$ \\
\hline & $2009^{+}$ & $0.08(0.24)$ & $0.52(0.06)$ & $-0.08(0.28)$ & $0.48(0.07)$ & $0.54(0.06)$ & $0.50(0.07)$ & $0.53(0.06)$ \\
\hline & $2010^{+}$ & $-0.86(0.18)$ & $0.30(0.04)$ & $-1.0(0.22)$ & $0.27(0.04)$ & $0.32(0.04)$ & $0.29(0.04)$ & $0.30(0.04)$ \\
\hline & $2011^{+}$ & $-0.34(0.26)$ & $0.42(0.06)$ & $-0.54(0.31)$ & $0.37(0.07)$ & $0.42(0.06)$ & $0.40(0.07)$ & $0.42(0.06)$ \\
\hline & $2012^{+}$ & $-0.69(0.26)$ & $0.33(0.06)$ & $-0.84(0.29)$ & $0.30(0.06)$ & $0.35(0.06)$ & $0.32(0.06)$ & $0.34(0.06)$ \\
\hline & year*loc & na & na & $0.23(0.22)$ & na & na & na & na \\
\hline
\end{tabular}

tDetection estimates for years 2009-2012 represent the probability of at least one fecal pellet detected in a single 12 m-diameter plot, given that the patch is occupied. In years prior to 2009, detection estimates represent the probability that pellets are detected in a habitat patch, given that the patch is occupied.

or contractions) was adequate to permit inference about the neighborhood occupancy effect.

We explored these general relationships proposed by metapopulation theory using the Lower Keys system as a case study. Our models accounted for the proportion of a focal patch's neighborhood that was composed of rabbit habitat, allowing us to better estimate neighbor effects for both coastal and interior patches and under conditions of considerable habitat fragmentation in three distinct regions of the Lower Keys. Previous research on rabbit dispersal admitted substantial uncertainty regarding the effective distance from a focal patch used to define a neighborhood (Forys and Humphrey 1996, Faulhaber et al. 2006). Our model selection results supported an effective neighborhood size of approximately $1000 \mathrm{~m}$ from the edge of a habitat patch. Based on this distance, average neighborhood 
occupancy had a relatively large positive effect on colonization and was negatively related to extinction probability. Weighting the autologistic covariate by the proportion of habitat within a patch's neighborhood $(\eta)$ permitted the post hoc detection of a possible ecological threshold that may function as a driver of significant changes in estimated patch occupancy, especially for inland patches. Additionally, high extinction probability for small coastal patches appeared to be mitigated by a rescue effect when neighboring patches within the effective buffer distance had high average occupancy. Both interior and coastal sites demonstrated substantial increases in colonization rates with high average neighborhood occupancy.

Parameter estimates produced from the best-supported model conformed to our system-specific expectations in most cases. Based on the observations of wildlife managers, LKMR populations are considered to be relatively stable on Sugarloaf, Saddlebunch and Boca Chica Keys (SBC) and slowly declining on Big Pine Key (BPK), especially in coastal patches, while patches on GAP islands are believed to be unpopulated. Our summary of patch-based dynamics using vital rates (Eq. 1) supported these observations, with higher average occupancy and population stability on SBC relative to BPK, and revealed a larger difference between coastal vs. interior occupancy on BPK relative to SBC (Fig. 4). GAP Keys had significantly lower colonization and higher extinction rates relative to the other metapopulations, but estimates were much more imprecise (high standard errors) due to a lack of data from which to calculate estimates (i.e. few colonization events observed and virtually no possibility of extinctions with near-zero occupancy). Estimates regarding the effect of patch size also conformed to expectations, with larger patches experiencing higher colonization and lower extinction rates.

Our findings that interior patches experienced higher average occupancy than coastal sites agreed with estimates based on a single-season model (Eaton et al. 2011) despite the fact that the single-season analysis led us to hypothesize that the underlying mechanisms might include higher initial occupancy and higher colonization rates in interior patches. The application of multi-season models, however, offered evidence that coastal sites were subject to greater population turnover, with higher rates of both colonization and extinction relative to interior patches. Given that the supratidal saltmarsh-buttonwood zone is believed to be the most important habitat for breeding, forage and cover (Forys 1995, Forys and Humphrey 1996), high turnover rates in coastal patches may be a function of 'push disturbance' (sea-level rise) or increasing frequency of high-tide events resulting in unstable disturbance regimes for LKMR (US Fish and Wildlife Service 2007). Indeed, the data supported a trend over time for both extinction and colonization probabilities, with coastal sites experiencing more rapid increases in extinction rates in recent years. Extirpation of rabbits in coastal patches may have been somewhat moderated by continued colonization of this higher quality habitat. We estimated that changes in turnover dynamics progressed more slowly over the study period for interior patches than in coastal patches, further suggesting that inland sites may serve as refugia under changing climatic conditions.
Coastal habitats have changed greatly over the past century with landward shifts of lower-diversity, intertidal communities into both supratidal zones and the lowerlying, higher-diversity perimeters of upland areas. Such incursions include mangroves (Rhizophora mangle, Laguncularia racemosa, Avicennia germinans) and buttonwood (Conocarpus erectus) into cordgrass (Spartina spartinae) marshes and low-lying uplands (e.g. slash pine, Pinus elliotii var densa) (Ross et al. 1994, 2009, US Fish and Wildlife Service 2007). Coastal habitats are more susceptible to detrimental impacts of high-tide events, the more gradual push of sea level rise and synergistic effects between the two (Faulhaber et al. 2007, US Fish and Wildlife Service 2007, Ross et al. 2009). Other researchers have suggested previously that some upland habitats may provide potential refuge during stochastic and regular (seasonal) flooding events (Faulhaber et al. 2008). Although tidal events are occurring more frequently and may be amplified by sea-level rise (Ross et al. 2009), impacts on interior freshwater wetlands have been less pronounced due to the lower likelihood that a given storm surge will result in saltwater intrusion of interior habitats. For these reasons, we consider the freshwater (interior) systems to have been more stable in recent decades for LKMR, even though they may not be necessarily more productive. Continued sea-level rise will lead to further modification of vegetation communities and other features of intertidal and supratidal systems, subjecting LKMR to even higher turnover rates in coastal patches.

Although historic LKMR survey methods are assumed to have been standardized within individual studies (Supplementary material Appendix 4 Table A4.3, H1-H5), we demonstrated that variation in detection probabilities existed even under standardized survey methods. Possible reasons include variation in searching ability among multiple observers, changes in observation (e.g. time of day) or field conditions (e.g. climate, browse quality effects on pellet persistence), or habitat characteristics not included as covariates in our detection model. In contrast with our earlier, single-season analysis (Eaton et al. 2011), we found some evidence that habitat type (the covariate 'location') induced variation in detection probability. As no information on vegetation type was available from historical records, we were unable to include a more detailed vegetation classification as a covariate for detection. We recognize that differences in detection probability may have been insensitive to our simple binary surrogate for habitat type and refuge personnel have begun to characterize patches by cover types and other variables that may be important for understanding both ecological and observation processes for future analyses.

It is important to distinguish the meaning of detection probability for historical surveys and the modern survey protocol (i.e. spatially replicated plots used from 2009-2012). In modern surveys, detection probability estimates refer to the probability that sign of LKMR is detected on a single $12 \mathrm{~m}$-diameter sample plot, given that the patch in which the plot is located is occupied by the species. The intent of historic surveys, even those that used regularly-spaced grid plots, was to determine with certainty the presence or absence 
of LKMRs in a habitat patch. Thus, previous workers attempted to perform complete searches, as there was no mechanism for dealing with nondetection. Even though such complete searches seldom resulted in detection probabilities approaching 1.0, they did produce sampling that was roughly proportional to patch size (i.e. more sampling in larger patches), allowing us to take advantage of the longterm data set. Inferences about detection probability generated from historic surveys applied to the entire patch, rather than to a single sample plot within a patch.

\section{Modeling approach}

The primary objectives for our analyses were to first test general hypotheses from metapopulation theory and then to test specific ideas about LMKR populations. In the course of addressing these objectives, we found it necessary to develop a method to incorporate spatial realism when fitting metapopulation models to data from our natural system. We believed that this would be especially important for inference about occupancy dynamics for LKMR, a species with limited dispersal abilities occupying a highly fragmented environment. Here, we built on the recent advances in autologistic models by Bled et al. (2011) and Yackulic et al. (2012), who developed models to focus on the mechanistic processes underlying species invasions.

Since their introduction, metapopulation and, later, occupancy models have been fundamentally concerned with space and the movement of individuals. Both attribute a strong emphasis to local extinction and immigration/colonization as determinants of patch dynamics but, until recently, occupancy models have been unable to explicitly consider the influence of the occupancy status of neighboring sites in a more fully mechanistic approach. Rather, they have relied on the presence of potential habitat in the neighborhood, or other indices of land cover structure, extent or type, as substitutes for the presence of potential colonizers. Additionally, a major difficulty with modeling metapopulation processes is that the occupancy status of a neighboring site cannot be treated as a standard covariate, but itself must be estimated. Following the examples of recently developed autologistic neighborhood models, we have incorporated further realism into spatial metapopulation models and have provided a method that offers greater flexibility in defining 'neighborhood' in relevant ways and fitting empirical data to test for autologistic effects. Our specification of annual, weighted average neighborhood occupancy was written to allow individual neighbor patches to vary in their ability to influence the vital rates (colonization and extinction) of a focal patch. This approach permits ample flexibility in testing hypotheses related to dispersal barriers, relative patch size, quality or distance, or other factors of potential biological relevance. We added additional flexibility to the autologistic term by considering the proportion of non-habitat in the neighborhood of a focal patch from which colonizers are not likely to originate. Our autologistic covariate, the product of the weighted neighborhood patch occupancy $\left(\hat{\bar{\Psi}}_{i, t}^{N_{i}}\right)$ and proportion of neighborhood actually comprising habitat $\left(\eta_{i}\right)$, permits a great deal of spatial realism to be incorporated into multi-season occupancy models. Possible extensions of this type of autologistic modeling could include addressing multiple states (MacKenzie et al. 2009) and/or false positive detections (Royle and Link 2006, Miller et al. 2011, 2013).

Rather than reject historical survey data that were collected without regard to the reality of imperfect detections, we included them in our analysis believing that models could produce unbiased maximum likelihood estimates under restricted parameterizations. We anticipated that modeling time-dependent variation in vital rate parameters (extinction and colonization) and detection probability would not be possible due to parameter non-identifiability. However, a model that incorporated simplified descriptions of temporal dynamics (trends in extinction and colonization) was well supported when fit to our data. Although this finding is not of direct relevance to managers, it should be encouraging to realize that historical data may be of value for such analyses.

\section{LKMR conservation}

Our findings have significant conservation and management implications specific to the LKMR. The recovery plan for this species stipulates counts of occupied sites as a means for determining species status and distribution. Due to the small population size of the LKMR, this metric will be highly sensitive to incorrect classifications of patch occupancy and, therefore, accounting for the effects of imperfect detection when estimating occupancy is valuable for monitoring recovery efforts. Further, historical selection of LKMR patches for monitoring was often based on previous detections (i.e. known occupancy) with patches possibly being excluded from subsequent survey efforts following a series of nondetections. More efficient monitoring decisions can be made by considering the effects of potential non-detections in addition to patch covariates (size, location and neighborhood configuration and occupancy) that we found to be important determinants of occupancy.

The best-supported model of occupancy dynamics suggested that patches in coastal areas experienced higher turnover (higher probabilities of colonization and extinction) than inland patches and that disturbance from sealevel rise and changing storm and vegetation dynamics may already be further destabilizing coastal patches. Inland patches may, however, function as refugia and a source for colonization of coastal patches following local extinctions. Finally, inference on effective neighborhood size, in conjunction with these other findings, provides managers with guidelines on spatial conservation planning for recovery efforts of the LKMR. The existence of a possible threshold in the proportion of habitat within a patch's neighborhood that may strongly influence metapopulation processes should be considered when prioritizing patches for habitat restoration, rabbit translocation or predator control.

Acknowledgements - We thank C. Yackulic, E. Crone, D. Doak, E. Cooch a for commenting on earlier versions of this manuscript. Kate Cardenas was instrumental in conducting the modern surveys. Any use of trade, product, or firm names is for descriptive purposes only and does not imply endorsement by the U.S. Government. The findings and conclusions in this article are 
those of the author(s) and do not necessarily represent the views of the U.S. Fish and Wildlife Service.

\section{References}

Bender, D. J. and Fahrig, L. 2005. Matrix structure obscures the relationship between interpatch movement and patch size and isolation. - Ecology 86: 1023-1033.

Bled, F. et al. 2011. Hierarchical modeling of an invasive spread: the Eurasian collared-dove Streptopelia decaocto in the United States. - Ecol. Appl. 21: 290-302.

Brown, J. H. and Kodric-Brown, A. 1977. Turnover rates in insular biogeography - effect of immigration on extinction. - Ecology 58: 445-449.

Brownie, C. et al. 1993. Capture-recapture studies for multiple strata including non-Markovian transition probabilities. - Biometrics 49: 1173-1187.

Caughley, G. 1994. Directions in conservation biology. - J. Anim. Ecol. 63: 215-244.

Clinchy, M. et al. 2002. Pattern does not equal process: what does patch occupancy really tell us about metapopulation dynamics? - Am. Nat. 159: 351-62.

Cook, W. et al. 2004. Is the matrix really inhospitable? Vole runway distribution in an experimentally fragmented landscape. - Oikos 104: 5-14.

Crone, E. E. et al. 2001. Ecological influences on the dynamics of a field vole metapopulation. - Ecology 82: 831-843.

Crooks, K. R. et al. 2001. Extinction and colonization of birds on habitat islands. - Conserv. Biol. 15: 159-172.

Crouse, A. L. et al. 2009. Population structure of the Lower Keys marsh rabbit as determined by mitochondrial DNA analysis. - J. Wildlife Manage. 73: 362-367.

Dail, D. and Madsen, L. 2011. Models for estimating abundance from repeated counts of an open metapopulation. - Biometrics 67: $577-87$.

Eaton, M. J. et al. 2011. Spatial patch occupancy patterns of the Lower Keys marsh rabbit. - J. Wildlife Manage. 75: 1186-1193.

Ellner, S. and Fussmann, G. 2003. Effects of successional dynamics on metapopulation persistence. - Ecology 84: 882-889.

Epps, C. W. et al. 2007. Optimizing dispersal and corridor models using landscape genetics. - J. Appl. Ecol. 44: 714-724.

Faulhaber, C. A. 2003. Updated distribution and reintroduction of the Lower Keys marsh rabbit. - MS thesis, Texas A\&M Univ., College Station, TX, USA.

Faulhaber, C. A. et al. 2006. Reintroduction of Lower Keys marsh rabbits. - Wildlife Soc. Bull. 34: 1198-1202.

Faulhaber, C. A. et al. 2007. Updated distribution of the Lower Keys marsh rabbit. - J. Wildlife Manage. 71: 208-212.

Faulhaber, C. A. et al. 2008. Diurnal habitat use by Lower Keys marsh rabbits. - J. Wildlife Manage. 72: 1161-1167.

Forys, E. A. 1995. Metapopulations of marsh rabbits: a population viability analysis of the Lower Keys marsh rabbit (Sylvilagus palustris hefneri). - PhD thesis, Univ. of Florida, Gainesville, FL, USA.

Forys, E. A. and Humphrey, S. R. 1996. Home range and movements of the Lower Keys marsh rabbit in a highly fragmented habitat. - J. Mammal. 77: 1042-1048.

Forys, E. A. and Humphrey, S. R. 1999. Use of population viability analysis to evaluate management the options for the endangered Lower Keys marsh rabbit. - J. Fish Wildlife Manage. 63: 251-260.

Fujiwara, M. and Caswell, H. 2002. A general approach to temporary emigration in mark-recapture analysis. - Ecology 83: 3266-3275.

Hanski, I. 1992. Inferences from ecological incidence functions. - Am. Nat. 193: 657-662.
Hanski, I. 1994. A practical model of metapopulation dynamics. - J. Anim. Ecol. 63: 151-162.

Hanski, I. 1998. Metapopulation dynamics. - Nature 396: 41-49.

Hanski, I. and Simberloff, D. 1997. The metapopulation approach, its history, conceptual domain, and application to conservation. - In: Hanski, I. A. and Gilpin, M. E. (eds), Metapopulation biology. Academic Press, pp. 5-26.

Hines, J. E. 2006. PRESENCE4- Software to estimate patch occupancy and related parameters. - USGS-PWRC < www. mbr-pwrc.usgs.gov/software/presence.shtml $>$.

Howe, S. E. 1988. Lower Keys marsh rabbit status survey. - US Fish and Wildlife Service, Jacksonville Field Station. Jacksonville, FL, USA.

Joshi, P. K. et al. 2011. Assessing impact of forest landscape dynamics on migratory corridors: a case study of two protected areas in Himalayan foothills. - Biodivers. Conserv. 20: 3393-3411.

Kendall, W. L. and Nichols, J. D. 2002. Estimating state-transition probabilities for unobservable states using capture-recapture/ resighting data. - Ecology 83: 3276-3284.

Kennedy, C. M. et al. 2011. Landscape matrix mediates occupancy dynamics of Neotropical avian insectivores. - Ecol. Appl. 21: $1837-50$.

Lazell, J. D. 1989. Wildlife of the Florida Keys: a natural history. - Island Press.

Lebreton, J.-D. et al. 2009. Modeling individual animal histories with multistate capture-recapture models. - Adv. Ecol. Res. 41: 87-173.

Lele, S. R. et al. 2012. Dealing with detection error in site occupancy surveys: what can we do with a single survey? - J. Plant Ecol. 5: 22-31.

Levins, R. 1969. Some demographic and genetic consequences of environmental heterogeneity for biological control. - Bull. Entomol. Soc. Am. 15: 237-240.

Levins, R. 1970. Extinction. - In: Gerstenhaber, M. (ed.), Some mathematical problems in biology. Am. Math. Soc., pp. 77-107.

Lookingbill, T. R. et al. 2010. Combining a dispersal model with network theory to assess habitat connectivity. - Ecol. Appl. 20: 427-41.

MacKenzie, D. I. et al. 2003. Estimating site occupancy, colonization, and local extinction when a species is detected imperfectly. - Ecology 84: 2200-2207.

MacKenzie, D. I. et al. 2006. Occupancy estimation and modeling: inferring patterns and dynamics of species occupancy. - Elsevier.

MacKenzie, D. I. et al. 2009. Modeling species occurrence dynamics with multiple states and imperfect detection. - Ecology 90: 823-35.

MacKenzie, D. I. et al. 2011. An integrated model of habitat and species occurrence dynamics. - Meth. Ecol. Evol. 2: 612-622.

McRae, B. H. et al. 2008. Using circuit theory to model connectivity in ecology, evolution and conservation. - Ecology 89: $2712-2724$.

Miller, D. et al. 2011. Improving occupancy estimation when two types of observational error occur: non-detection and species misidentification. - Ecology 92: 1422-1428.

Miller, D. A. W. et al. 2012. Joint estimation of habitat dynamics and species interactions: disturbance reduces co-occurrence of non-native predators with an endangered toad. - J. Anim. Ecol. 81: 1288-1297.

Miller, D. A. W. et al. 2013. Determining occurrence dynamics when false positives occur: estimating the range dynamics of wolves from Public Service data. - PLoS ONE 8: e65808.

Moilanen, A. 1999. Patch occupancy models of metapopulation dynamics: efficient parameter estimation using implicit statistical inference. - Ecology 80: 1031-1043.

Moilanen, A. and Nieminen, M. 2002. Simple connectivity measures in spatial ecology. - Ecology 83: 1131-1145.

Nicholson, E. and Ovaskainen, O. 2009. Conservation prioritization using metapopulation models. - In: Moilanen, A. et al. (eds), 
Spatial conservation prioritization: quantitative methods and computational tools. Oxford Univ. Press, pp. 110-121.

Pellet, J. et al. 2007. An empirical evaluation of the area and isolation paradigm of metapopulation dynamics. - Biol. Conserv. 136: 483-495.

Prugh, L. R. et al. 2008. Effect of habitat area and isolation on fragmented animal populations. - Proc. Natl Acad. Sci. USA 105: 20770-207705.

Robles, H. and Ciudad, C. 2012. Influence of habitat quality, population size, patch size and connectivity on patchoccupancy dynamics of the middle spotted woodpecker. - Conserv. Biol. 26: 284-293.

Ross, M. S. et al. 1994. Sea-level rise and the reduction in pine forests in the Florida Keys. - Ecol. Appl. 4: 144-156.

Ross, M. S. et al. 2009. Disturbance and the rising tide: the challenge of biodiversity management on low-island ecosystems. - Front. Ecol. Environ. 7: 471-478.

Royle, J. A. and Link, W. A. 2006. Generalized site occupancy models allowing for false positive and false negative errors. - Ecology 87: 835-841.

Royle, J. A. and Dorazio, R. M. 2008. Hierarchical modeling and inference in ecology. - Elsevier-Academic Press.

Royle, J. A. et al. 2013. Spatial capture-recapture models for jointly estimating population density and landscape connectivity. - Ecology 94: 287-294.

Sanderlin, J. S. et al. 2012. On valuing patches: estimating contributions to metapopulation growth with reverse-time capture-recapture modelling. - Proc. R. Soc. B 279: 480-488.

Schmidt, P. M. 2009. Metapopulation ecology and recovery of the endangered Lower Key marsh rabbit. - PhD thesis, Texas A\&M Univ., College Station, TX, USA.

Schmidt, J. A. et al. 2011a. Population estimation and monitoring of an endangered lagomorph. - J. Wildlife Manage. 75: 151-158.

Schmidt, P. M. et al. 2011b. Influence of patch, habitat, and landscape characteristics on patterns of Lower Keys marsh rabbit occurrence following Hurricane Wilma. - Landscape Ecol. 26: 1419-1431.

Supplementary material (available as Appendix oik-01008 at $<$ www.oikosjournal.org/readers/appendix $>$ ). Appendix $1-5$, Fig. A1, supplement ( $R$ code and data files for simulation analysis).
Schmidt, J. A. et al. 2012. Impacts of a half century of sea-level rise and development on an endangered mammal. - Global Chang. Biol. 18: 3536-3542.

Sutherland, C. et al. 2012. Multi-scale processes in metapopulations: contributions of stage structure, rescue effect and correlated extinctions. - Ecology 93: 2465-2473.

Tursi, R. M. et al. 2012. Taxonomy versus phylogeny: evolutionary history of marsh rabbits without hopping to conclusions. - Divers. Distrib. 19: 120-133.

US Fish and Wildlife Service 1990. Endangered and threatened wildlife and plants; endangered status for the Lower Keys rabbit and threatened status for the squirrel chimney cave shrimp. - Fed. Regist. 55: 25588-25591.

US Fish and Wildlife Service 1999. Lower Keys rabbit, Sylvilagus palustris hefneri. - In: South Florida multi-species recovery plan. pp. 151-172.

US Fish and Wildlife Service 2007. Lower Keys marsh rabbit (Sylvilagus palustris hefneri). - 5-Year Rev. Summ. Eval.: $1-30$.

Vuilleumier, S. et al. 2007. How patch configuration affects the impact of disturbances on metapopulation persistence. - Theor. Popul. Biol. 72: 77-85.

Wilcox, C. et al. 2006. The role of habitat disturbance and recovery in metapopulation persistence. - Ecology 87: $855-863$.

Winfree, R. et al. 2005. Testing simple indices of habitat proximity. - Am. Nat. 165: 707-17.

Wright, S. 1931. Evolution in Mendelian populations. - Genetics 16: $97-159$.

Wright, S. 1940. Breeding structure of populations in relation to speciation. - Am. Nat. 74: 232-248.

Yackulic, C. B. et al. 2012. Neighborhood and habitat effects on vital rates: expansion of the barred owl in the Oregon coast ranges. - Ecology 93: 1953-1966.

Yackulic, C. B. et al. 2014. The roles of competition and habitat in the dynamics of populations and species distributions. - Ecology 95: 265-279. 